Author affiliations appear at the end of this article.

Published online ahead of print at www.jco.org on July 7, 2014.

Authors' disclosures of potential conflicts of interest and author contributions are found at the end of this article.

Corresponding author: Andrea Maurichi, MD, Department of Surgery, Fondazione Istituto Di Ricovero e Cura a Carattere Scientifico, Istituto Nazionale dei Tumori, Via Giacomo Venezian 1, 20133 Milan, Italy; e-mail: andrea.maurichi@ istitutotumori.mi.it.

(C) 2014 by American Society of Clinical Oncology

0732-183X/14/3223w-2479w/\$20.00

DOI: $10.1200 / J C O .2013 .54 .2340$

\title{
Prediction of Survival in Patients With Thin Melanoma: Results From a Multi-Institution Study
}

Andrea Maurichi, Rosalba Miceli, Tiziana Camerini, Luigi Mariani, Roberto Patuzzo, Roberta Ruggeri, Gianfranco Gallino, Elena Tolomio, Gabrina Tragni, Barbara Valeri, Andrea Anichini, Roberta Mortarini, Daniele Moglia, Giovanni Pellacani, Sara Bassoli, Caterina Longo, Pietro Quaglino, Nicola Pimpinelli, Lorenzo Borgognoni, Daniele Bergamaschi, Catherine Harwood, Odysseas Zoras, and Mario Santinami

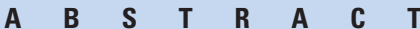

\section{Purpose}

Cutaneous melanoma incidence is increasing. Most new cases are thin $(\leq 1 \mathrm{~mm})$ with favorable prognoses, but survival is nonetheless variable. Our aim was to investigate new prognostic factors and construct a nomogram for predicting survival in individual patients.

\section{Patients and Methods}

Data from 2,243 patients with thin melanoma were retrieved from prospectively maintained databases at six centers. Kaplan-Meier survival and crude cumulative incidences of recurrence were estimated, and competing risks were taken into account. Multivariable Cox regression was used to investigate survival predictors.

\section{Results}

Median follow-up was 124 months (interquartile range, 106 to 157 months); 12-year overall survival was $85.3 \%(95 \% \mathrm{Cl}, 83.4 \%$ to $87.2 \%)$. Median times to local, regional, and distant recurrence were 79, 78, and 107 months, respectively. Relapse was significantly related to age, Breslow thickness, mitotic rate (MR), ulceration, lymphovascular invasion (LVI), and regression; incidence was lower and subgroup differences were less marked for distant metastasis than for regional relapse. The worst prognosis categories were age older than 60 years, Breslow thickness more than $0.75 \mathrm{~mm}, \mathrm{MR} \geq 1$, presence of ulceration, presence of $L V I$, and regression $\geq 50 \%$. Breslow thickness more than 0.75 $\mathrm{mm}, \mathrm{MR} \geq 1$, presence of ulceration, and LVI (all $P=.001$ ) were significantly associated with sentinel node positivity. Age, MR, ulceration, LVI, regression, and sentinel node status were independent predictors of survival and were used to construct a nomogram to predict 12-year overall survival. The nomogram was well calibrated and had good discriminative ability (adjusted Harrell C statistic, 0.88).

\section{Conclusion}

Our findings suggest including LVI and regression as new prognostic factors in the melanoma staging system. The nomogram appears useful for risk stratification in clinical management and for recruiting patients to clinical trials.

\section{J Clin Oncol 32:2479-2485. (C) 2014 by American Society of Clinical Oncology}

\section{INTRODUCTION}

The incidence of cutaneous melanoma is increasing. ${ }^{1}$ Most new cases are diagnosed when thin (Breslow thickness $\leq 1 \mathrm{~mm})^{2}$ and have favorable prognoses. However, the 2009 American Joint Committee on Cancer (AJCC) emphasized the variability ( $85 \%$ to $99 \%$ ) of 10 -year survival for thin melanoma. ${ }^{3}$ It is important therefore to identify factors that influence prognosis.

Although thickness has long been recognized as an important prognostic factor for primary localized melanoma, ${ }^{4}$ various other factors have been proposed as prognostic and suggested as stage indicators. ${ }^{5}$ The current AJCC classification ${ }^{3}$ recognizes two tumor-associated factors for thin melanomas: mitotic rate (MR) and ulceration. Low-risk (T1a) melanomas are nonulcerated, with an MR of less than one mitosis per square millimeter; higher-risk (T1b) melanomas have an MR of one or more mitoses per square millimeter, ulceration, or both. The Committee noted that regional node metastasis was also an important prognostic factor in early-stage melanoma and recommended sentinel node biopsy (SNB) in selected T1b patients ${ }^{3}$ but did not have sufficient data to assess the risk of occult nodal micrometastases in $\mathrm{T} 1$ patients.

Several studies have attempted to define the role of SNB in thin melanoma and identify predictors of sentinel node (SN) positivity, but findings have been discordant. ${ }^{6-8}$ Recent guidelines, ${ }^{9,10}$ also 
noting the lack of evidence to support routine SNB in T1 patients, state that SNB should be considered for selected high-risk patients.

These considerations highlight the variable risks associated with T1 melanomas and the need to identify characteristics for reliable risk stratification. The aim of this study was to identify determinants of survival and predictors of SN status in a large multicentric series of patients with thin melanoma and construct a nomogram predicting survival.

\section{PATIENTS AND METHODS}

All patients diagnosed and treated for a single thin (Breslow thickness $1.00 \mathrm{~mm}$ or less) primary melanoma from 1996 through 2004 at six European centers were considered. Clinicopathologic data were retrieved from prospectively maintained databases. Patients with satellites or metastases at diagnosis or history of other cancer were excluded. Information retrieved comprised age, sex, site (head and neck, trunk, limbs), Breslow thickness, MR, ulceration, Clark level, presence or absence of lymphovascular invasion (LVI), regression, and tumor-infiltrating lymphocytes (TILs). MR was expressed as number of mitoses per square millimeter. TILs were classified as brisk, nonbrisk, or absent. Regression was characterized as absent, partial $(<50 \%$ of the entire primary lesion), or extensive ( $\geq 50 \%$ ). LVI was defined as the presence of melanoma cells within lymphatic or blood vessels.

After excluding 86 patients lost immediately after discharge and 42 patients with missing data, 2,243 patients were included. All slides were reviewed independently by three pathologists (G.T., B.V., and C.L.) with disagreements resolved by discussion.

Treatment consisted of diagnostic excision with 1-to 2-mm margins followed by wider excision to achieve histologically confirmed 1-cm margins in healthy tissue. SNB was offered to high-risk patients, for which criteria were Breslow thickness 0.75 to $1.00 \mathrm{~mm}$, MR one or more mitoses per square millimeter, presence of ulceration, presence of LVI, Clark level IV or V, and extensive regression. The benefits and risks of SNB were discussed with patients. Some patients asked for and received SNB, although the risk of occult nodal metastasis was low. All patients with a positive $\mathrm{SN}$ were urged to undergo node dissection.

Recurrences subsequent to definitive surgery were classified as local if they developed within the primary scar, regional if they were in-transit dermal or subcutaneous metastases or in lymph nodes, and distant if they were nonregional skin, nodal, or visceral metastases. All skin and node recurrences were confirmed histologically.

The $\chi^{2}$ or Wilcoxon-Mann-Whitney test was used to determine significant differences between stage T1a and T1b. Multivariable binary logistic modeling was used to explore propensity to perform SNB according to year of diagnosis, sex, age, tumor site, Breslow thickness, MR, ulceration, LVI, Clark level, TILs, and regression. The response variable was zero if SNB was not performed and 1 if it was performed.

In patients receiving $\mathrm{SNB}$, the Wilcoxon-Mann-Whitney test (year of diagnosis, age, Breslow thickness) or $\chi^{2}$ test (other variables) assessed differences in the distribution of these variables between SN-negative and SNpositive groups.

The main study end point was overall survival (OS) calculated from date of surgery for primary melanoma to date of death as a result of all causes or censored at date of last follow-up in living patients. OS curves were estimated by using the Kaplan-Meier method, and the log-rank test was used to compare subgroups. Additional end points were (crude cumulative incidences of) regional relapse and distant metastasis, analyzed in a competing risks framework $^{11}$; time was calculated from date of definitive surgery to event date and was censored at date of last follow-up in event-free patients. For regional relapse, competing events were distant metastasis, death as a result of an unrelated cause, local relapse, or second malignancy, whichever occurred first. For distant metastasis, competing events were regional relapse, death as a result of an unrelated cause, local relapse, and second malignancy. ${ }^{12}$ Because cumulative incidences did not reach $50 \%$, we noted values at maximum observation time and halved them: times at which half the maximum cumulative incidence occurred were considered median times to events.

Multivariable Cox modeling was used to analyze OS. The proportional hazards assumption was checked by using tests based on scaled Schoenfeld residuals. ${ }^{13}$ The covariates age, Breslow thickness, MR, ulceration, LVI, regression, SNB status, and SN status were investigated as prognostic factors. SNB and SN status were initially represented by a three-level covariate (SNB not done, SNB done/SN negative, SNB done/SN positive). However, the proportional hazards assumption did not hold in that the hazard ratio for SNB not done versus SNB done/SN negative tended to increase after 8 years. Use of SNB was therefore modeled as a stratification factor, and its interaction with $\mathrm{SN}$ status was included as a covariate; the latter made it possible to estimate the prognostic effect of $\mathrm{SN}$-positive versus $\mathrm{SN}$-negative disease, conditional on SNB. SNB use did not interact significantly with other prognostic factors, so no other interaction terms were included. A backward procedure based on the Akaike information criterion ${ }^{14}$ was used to select covariates.

The nomogram to predict 12-year OS probability was developed from the final Cox model. Nomogram performance was assessed by calibration plot as an indicator of internal calibration and by the Harrell C statistic as a measure of discriminative ability. ${ }^{15}$ The Harrell C statistic corresponds to the area under the receiver operating characteristic curve; values 0.5 and 1 , respectively, indicate lack of discriminative ability and perfect discriminative ability. A bootstrap procedure ${ }^{16}$ was adopted to adjust the $\mathrm{C}$ statistic estimate for the optimism implicit in the use of sample data for model fitting and variable selection.

In the multivariable models, year of diagnosis, age, and Breslow thickness were continuous variables using three-knot restricted cubic splines. ${ }^{17} \mathrm{Re}-$ stricted cubic spline modeling has the advantage of avoiding categorization and use of cutoffs (necessary to estimate Kaplan-Meier and crude cumulative incidence curves) and of obtaining a flexible fit allowing the effects of continuous variables not to be the same in every part of the range. Thus, the covariate values presented in the Cox model tables do not define categories but are exact values (quartiles of the variable distribution). All categorical covariates were modeled by using dummy variables. The analyses were carried out with SAS (SAS Institute, Cary, NC) and R software (http://www.r-project.org/).

\section{RESULTS}

\section{Patient and Disease Characteristics}

Characteristics of the 2,243 patients, by stage, are summarized in Table 1. Stage IA and IB patients did not differ regarding sex, age, site, or presence of TILs. Of the T1a lesions, $45.2 \%$ were $\leq 0.50 \mathrm{~mm}$ compared with $26.3 \%$ of T1b lesions, and $24.9 \%$ of T1a lesions were more than $0.75 \mathrm{~mm}$ compared with $43.7 \%$ of T1b lesions. Among the 1,115 T1b patients, $982(88.1 \%)$ had $\mathrm{MR} \geq 1$ and $530(47.5 \%)$ had ulceration. LVI was present in $19.1 \%$ of Tla patients and was present in $43.6 \%$ of T1b patients. T1a patients were less likely than T1b patients to have regression: less than $50 \%$ regression, $17.4 \%$ versus $21.9 \%$; $\geq 50 \%$ regression, $10.3 \%$ versus $18.0 \%$.

\section{$S N B$}

Overall, 794 patients (35.4\%) received SNB (Table 2). The percentage of patients undergoing SNB (propensity) tended to decrease with advancing age (from $36 \%$ to $28.8 \%$ ). As expected, patients with worse prognostic factors underwent SNB more often. Thus, propensity to undergo SNB increased with increasing Breslow thickness ( $11.3 \%$ for $\leq 0.50 \mathrm{~mm} ; 60.2 \%$ for $>0.75 \mathrm{~mm}$ ) and increasing $\mathrm{MR}$ (from $28.1 \%$ to $44.8 \%$ ).

Multivariable analysis showed that characteristics significantly associated with propensity to undergo SNB were age, Breslow thickness, ulceration, and LVI (all $P<.001$ ). Sixty-eight patients $(8.6 \%)$ 


\begin{tabular}{|c|c|c|c|c|c|c|c|}
\hline \multirow[b]{2}{*}{ Characteristic } & \multicolumn{2}{|c|}{ Stage T1a } & \multicolumn{2}{|c|}{ Stage T1b } & \multirow[b]{2}{*}{$P^{*}$} & \multicolumn{2}{|c|}{$\begin{array}{l}\text { Whole } \\
\text { Series }\end{array}$} \\
\hline & No. & $\%$ & No. & $\%$ & & No. & $\%$ \\
\hline Total No. of patients & 1,128 & 50.3 & 1,115 & 49.7 & NA & 2,243 & 100.0 \\
\hline Year of diagnosis & & & & & $<.001$ & & \\
\hline 1996-1998 & 220 & 19.5 & 257 & 23.0 & & 477 & 21.3 \\
\hline $1999-2000$ & 228 & 20.2 & 177 & 15.9 & & 405 & 18.1 \\
\hline 2001-2002 & 217 & 19.2 & 301 & 27.0 & & 518 & 23.1 \\
\hline $2003-2004$ & 463 & 41.0 & 380 & 34.1 & & 843 & 37.6 \\
\hline Sex & & & & & .930 & & \\
\hline Female & 612 & 54.3 & 608 & 54.5 & & 1,220 & 54.4 \\
\hline Male & 516 & 45.7 & 507 & 45.5 & & 1,023 & 45.6 \\
\hline Age, years & & & & & .767 & & \\
\hline Median & & 3.5 & 43 & 3 & & 43 & 3 \\
\hline IQR & $36-$ & -53 & $36.5-$ & -52 & & $36-$ & -52 \\
\hline$\leq 40$ & 445 & 39.5 & 424 & 38.0 & & 869 & 38.7 \\
\hline$>40$ to $\leq 50$ & 323 & 28.6 & 315 & 28.3 & & 638 & 28.4 \\
\hline$>50$ to $\leq 60$ & 228 & 20.2 & 236 & 21.2 & & 464 & 20.7 \\
\hline$>60$ to $\leq 70$ & 79 & 7.0 & 89 & 8.0 & & 168 & 7.5 \\
\hline$>70$ & 53 & 4.7 & 51 & 4.6 & & 104 & 4.6 \\
\hline Site & & & & & .966 & & \\
\hline Head and neck & 227 & 20.1 & 223 & 20.0 & & 450 & 20.1 \\
\hline Trunk & 470 & 41.7 & 460 & 41.3 & & 930 & 41.5 \\
\hline Upper or lower limbs & 431 & 38.2 & 432 & 38.7 & & 863 & 38.5 \\
\hline Breslow thickness, mm & & & & & $<.001$ & & \\
\hline Median & & 52 & 0.7 & 71 & & 0.6 & 63 \\
\hline IQR & $0.33-$ & -0.72 & $0.50-0$ & 0.79 & & $0.41-$ & -0.78 \\
\hline$\leq 0.50$ & 510 & 45.2 & 293 & 26.3 & & 803 & 35.8 \\
\hline$>0.50$ to $\leq 0.75$ & 337 & 29.9 & 335 & 30.0 & & 672 & 30.0 \\
\hline$>0.75$ to $\leq 1$ & 281 & 24.9 & 487 & 43.7 & & 768 & 34.2 \\
\hline Mitoses, No. per mm² & & & & & $<.001$ & & \\
\hline$<1$ & 1,128 & 100.0 & 133 & 11.9 & & 1,261 & 56.2 \\
\hline$\geq 1$ & 0 & 0.0 & 982 & 88.1 & & 982 & 43.8 \\
\hline Ulceration & & & & & $<.001$ & & \\
\hline Absent & 1,128 & 100.0 & 585 & 52.5 & & 1,713 & 76.4 \\
\hline Present & 0 & 0.0 & 530 & 47.5 & & 530 & 23.6 \\
\hline Lymphovascular invasion & & & & & $<.001$ & & \\
\hline Absent & 912 & 80.9 & 629 & 56.4 & & 1,541 & 68.7 \\
\hline Present & 216 & 19.1 & 486 & 43.6 & & 702 & 31.3 \\
\hline Clark level & & & & & $<.001$ & & \\
\hline II or III & 727 & 64.5 & 428 & 38.4 & & 1,155 & 51.5 \\
\hline IV & 401 & 35.5 & 687 & 61.6 & & 1,088 & 48.5 \\
\hline $\begin{array}{l}\text { Tumor-infiltrating } \\
\text { lymphocytes }\end{array}$ & & & & & .633 & & \\
\hline Absent & 587 & 52.0 & 568 & 50.9 & & 1,155 & 51.5 \\
\hline Present & 541 & 48.0 & 547 & 49.1 & & 1,088 & 48.5 \\
\hline Regression & & & & & $<.001$ & & \\
\hline Absent & 816 & 72.3 & 670 & 60.1 & & 1,486 & 66.3 \\
\hline Present $(<50 \%)$ & 196 & 17.4 & 244 & 21.9 & & 440 & 19.6 \\
\hline Present ( $\geq 50 \%$ ) & 116 & 10.3 & 201 & 18.0 & & 317 & 14.1 \\
\hline
\end{tabular}

were SN positive and underwent regional nodal dissection. Increasing Breslow thickness, high MR, ulceration, and LVI were significantly associated with SN positivity (Table 2). The low number of patients with a positive SN made reliable multivariable analysis of factors associated with SN positivity impossible.

\begin{tabular}{|c|c|c|c|c|c|c|}
\hline \multirow[b]{2}{*}{ Characteristic } & \multicolumn{2}{|c|}{$\begin{array}{l}\text { Undergoing } \\
\text { SNB }\end{array}$} & \multirow{2}{*}{$\begin{array}{l}\text { Percent } \\
\text { of Whole } \\
\text { Series** }\end{array}$} & \multicolumn{3}{|c|}{$\begin{array}{c}\text { Association } \\
\text { With SN } \\
\text { Positivity }\end{array}$} \\
\hline & No. & $\%$ & & No. & $\%$ & $P+$ \\
\hline Sex & & & & & & .999 \\
\hline Female & 414 & 52.1 & 33.9 & 35 & 8.5 & \\
\hline Male & 380 & 47.9 & 37.1 & 33 & 8.7 & \\
\hline Age, years & & & & & & .059 \\
\hline$\leq 40$ & 355 & 44.7 & 40.9 & 37 & 10.4 & \\
\hline$>40$ to $\leq 50$ & 188 & 23.7 & 29.5 & 16 & 8.5 & \\
\hline$>50$ to $\leq 60$ & 158 & 19.9 & 34.1 & 10 & 6.3 & \\
\hline$>60$ to $\leq 70$ & 63 & 7.9 & 37.5 & 4 & 6.3 & \\
\hline$>70$ & 30 & 3.8 & 28.8 & 1 & 3.3 & \\
\hline Site & & & & & & .993 \\
\hline Head and neck & 155 & 19.5 & 34.4 & 13 & 8.4 & \\
\hline Trunk & 322 & 40.6 & 34.6 & 28 & 8.7 & \\
\hline Upper or lower limbs & 317 & 39.9 & 36.7 & 27 & 8.5 & \\
\hline Breslow thickness, $\mathrm{mm}$ & & & & & & $<.001$ \\
\hline$\leq 0.50$ & 91 & 11.5 & 11.3 & 3 & 3.3 & \\
\hline$>0.50$ to $\leq 0.75$ & 241 & 30.4 & 35.9 & 11 & 4.6 & \\
\hline$>0.75$ & 462 & 58.2 & 60.2 & 54 & 11.7 & \\
\hline Mitoses, No. per $\mathrm{mm}^{2}$ & & & & & & $<.001$ \\
\hline$<1$ & 354 & 44.6 & 28.1 & 5 & 1.4 & \\
\hline$\geq 1$ & 440 & 55.4 & 44.8 & 63 & 14.3 & \\
\hline Ulceration & & & & & & $<.001$ \\
\hline Absent & 373 & 47.0 & 21.8 & 12 & 3.2 & \\
\hline Present & 421 & 53.0 & 79.4 & 56 & 13.3 & \\
\hline Lymphovascular invasion & & & & & & $<.001$ \\
\hline Absent & 436 & 54.9 & 28.3 & 24 & 5.5 & \\
\hline Present & 358 & 45.1 & 51.1 & 44 & 12.3 & \\
\hline Clark level & & & & & & .962 \\
\hline II or III & 370 & 46.6 & 32.0 & 31 & 8.4 & \\
\hline IV & 424 & 53.4 & 39.0 & 37 & 8.7 & \\
\hline Tumor-infiltrating lymphocytes & & & & & & .945 \\
\hline Absent & 353 & 44.5 & 30.6 & 31 & 8.8 & \\
\hline Present & 441 & 55.5 & 40.5 & 37 & 8.4 & \\
\hline Regression & & & & & & .212 \\
\hline Absent & 444 & 55.9 & 29.9 & 33 & 7.4 & \\
\hline Present (extent $<50 \%$ ) & 141 & 17.8 & 32.0 & 11 & 7.8 & \\
\hline Present (extent $\geq 50 \%$ ) & 209 & 26.3 & 65.9 & 24 & 11.5 & \\
\hline
\end{tabular}

Abbreviations: SN, sentinel node; SNB, sentinel node biopsy.

*Percentage of the total patients in each category.

tWilcoxon-Mann-Whitney test (age and Breslow thickness evaluated as continuous variables) and $\chi^{2}$ test (other variables).

\section{Relapse and Survival}

Median follow-up was 124 months (interquartile range, 106 to 157 months). There were nine local relapses, 169 regional appearances, and 70 distant metastases as first event. Fifty-five patients developed another malignancy as first event, and six died (first event) of causes unrelated to melanoma. Median times to local, regional, and distant first events were 79, 78, and 107 months, respectively.

Twelve-year estimates of crude cumulative incidences of regional relapse and distant metastasis, in relation to age, Breslow thickness, MR, ulceration, LVI, and regression, are provided in Table 3. Crude cumulative incidence of local relapse was not estimated for low numbers of events. All factors listed in Table 3 significantly (all $P<.001$ ) influenced crude cumulative incidences of regional relapse and distant metastases. In general, incidence was lower and subgroup differences 


\begin{tabular}{|c|c|c|c|c|c|c|}
\hline \multirow[b]{3}{*}{ Characteristic } & \multicolumn{4}{|c|}{ Crude Cumulative Incidence } & & \\
\hline & \multicolumn{2}{|c|}{ Regional Relapse } & \multicolumn{2}{|c|}{ Distant Metastasis } & \multicolumn{2}{|c|}{ OS } \\
\hline & $\%$ & $95 \% \mathrm{Cl}$ & $\%$ & $95 \% \mathrm{Cl}$ & $\%$ & $95 \% \mathrm{Cl}$ \\
\hline \multicolumn{7}{|l|}{ Age, years } \\
\hline$\leq 40$ & 1.2 & 0.6 to 2.3 & 2.1 & 1.2 to 3.5 & 96.8 & 95.3 to 98.2 \\
\hline$>40$ to $\leq 50$ & 6.5 & 4.7 to 8.9 & 4.6 & 2.6 to 8.2 & 88.0 & 84.3 to 92.0 \\
\hline$>50$ to $\leq 60$ & 7.7 & 5.5 to 10.7 & 6.2 & 4.1 to 9.4 & 82.9 & 78.7 to 87.4 \\
\hline$>60$ to $\leq 70$ & 38.6 & 30.4 to 49.0 & 10.8 & 5.9 to 19.7 & 40.3 & 31.0 to 52.5 \\
\hline$>70$ & 37.6 & 28.9 to 48.9 & 5.7 & 2.4 to 13.6 & 43.3 & 32.0 to 58.6 \\
\hline \multicolumn{7}{|l|}{ Breslow thickness, mm } \\
\hline$\leq 0.50$ & 1.1 & 0.5 to 2.3 & 1.9 & 1.0 to 3.7 & 96.8 & 95.2 to 98.5 \\
\hline$>0.50$ to $\leq 0.75$ & 3.3 & 2.1 to 5.0 & 3.2 & 1.9 to 5.5 & 92.2 & 89.6 to 94.8 \\
\hline$>0.75$ to $\leq 1$ & 19.8 & 17.1 to 23.0 & 7.0 & 5.2 to 9.4 & 70.8 & 67.0 to 74.9 \\
\hline \multicolumn{7}{|l|}{ Mitotic rate, No. per $\mathrm{mm}^{2}$} \\
\hline$<1$ & 1.8 & 1.1 to 2.8 & 2.4 & 1.5 to 3.7 & 95.3 & 93.8 to 96.8 \\
\hline$\geq 1$ & 16.5 & 14.2 to 19.2 & 6.2 & 4.7 to 8.3 & 74.7 & 71.4 to 78.2 \\
\hline \multicolumn{7}{|l|}{ Ulceration } \\
\hline Absent & 1.5 & 1.0 to 2.3 & 2.7 & 1.9 to 3.8 & 95.3 & 94.1 to 96.6 \\
\hline Present & 29.4 & 25.6 to 33.8 & 8.7 & 6.2 to 12.1 & 57.7 & 52.5 to 63.3 \\
\hline \multicolumn{7}{|l|}{ Lymphovascular invasion } \\
\hline Absent & 1.4 & 0.9 to 2.1 & 2.8 & 1.9 to 4.0 & 95.3 & 94.0 to 96.6 \\
\hline Present & 23.1 & 20.0 to 26.8 & 7.1 & 5.1 to 9.8 & 65.6 & 61.1 to 70.3 \\
\hline \multicolumn{7}{|l|}{ Regression } \\
\hline Absent & 1.8 & 1.3 to 2.7 & 3.4 & 2.4 to 4.9 & 94.1 & 92.6 to 95.7 \\
\hline Present $(<50 \%)$ & 1.5 & 0.7 to 3.2 & 2.9 & 1.6 to 5.2 & 94.1 & 91.5 to 96.8 \\
\hline Present $(\geq 50 \%)$ & 44.7 & 39.4 to 50.7 & 8.1 & 5.4 to 12.0 & 48.5 & 42.7 to 55.0 \\
\hline
\end{tabular}

Abbreviation: OS, overall survival.

less marked for distant metastasis than regional relapse. The poorer prognosis categories were age older than 60 years, Breslow thickness more than $0.75 \mathrm{~mm}, \mathrm{MR} \geq 1$, presence of ulceration, LVI, and regression $\geq 50 \%$.

Regarding mortality, 231 patients died of melanoma and nine of other causes; 12 -year OS was $85.3 \%$ (95\% CI, $83.4 \%$ to $87.2 \%$ ). Regional relapse and distant metastasis had a major impact on mortality: five of the nine who developed local relapse, 156 of the 169 who developed regional relapse, and all 70 patients who developed distant metastasis died of melanoma.

Table 3 also depicts (right-most two columns) 12-year OS estimates according to age, Breslow thickness, MR, ulceration, LVI, and regression. As for relapse, all factors investigated were significantly associated with OS, and prognostic trends were similar.

In the Cox model analysis of OS, SNB use was included as a stratification factor so that OS estimates could differ according to whether or not SNB was performed. The following were evaluated: age, Breslow thickness, MR, ulceration, LVI, regression, and SN status (conditional to receiving SNB). The results of the final Cox model are provided in Table 4. As a result of the selection procedure, Breslow thickness was excluded, and the categories "regression absent" and "regression present with extent less than 50\%" were fused. All remaining factors were highly significant predictors of OS. The exclusion of Breslow thickness from the model was the result of the strong association between Breslow thickness and the other factors retained in the model: greater thickness was associated with older age, $\mathrm{MR} \geq 1$, presence of ulceration, LVI, and regression $\geq 50 \%$.

The nomogram based on the final Cox model is shown in Figure 1. By using the nomogram, 12-year OS probability can be estimated from individual patient and tumor characteristics, conditional to SNB or not. Negative prognostic factors contributed points so that increasing total points were associated with increasingly worse prognosis. A more detailed description of nomogram use is given in the Figure 1 legend. Notably, a given point score implies worse survival for a patient not receiving SNB compared with a node-negative patient receiving SNB. The discrepancy, usually in the $5 \%$ to $10 \%$ range, may be explained by considering that unrecognized node-positive patients

Table 4. Multivariable Cox Regression Model of OS After Backward Selection of Variables

\begin{tabular}{|c|c|c|c|}
\hline Variable & $\mathrm{HR}$ & $95 \% \mathrm{Cl}$ & $P^{*}$ \\
\hline Age $52 \vee 36$ yearst & 3.88 & 2.69 to 5.59 & $<.001$ \\
\hline No. of mitoses per $\mathrm{mm}^{2} \geq 1 v<1$ & 1.58 & 1.06 to 2.37 & .026 \\
\hline Ulceration present $v$ absent & 3.81 & 2.51 to 5.80 & $<.001$ \\
\hline Lymphovascular invasion present $v$ absent & 1.81 & 1.24 to 2.65 & .002 \\
\hline Regression $\geq 50 \% \vee v$ absent or $<50 \%$ & 3.32 & 2.31 to 4.77 & $<.001$ \\
\hline SN status positive $v$ negative $\neq$ & 2.97 & 1.86 to 4.76 & $<.001$ \\
\hline
\end{tabular}

NOTE. Hazard ratio (HR) estimate is the risk of death for a given category or value compared with the reference category or value. HR $>1$ indicates greater risk than reference; $H R<1$ indicates lower risk than reference. The larger the $\mathrm{HR}$, the greater the association between the variable and risk of death. $\mathrm{Cls}$ that do not include the value of one indicate a significant difference for the category compared with reference.

Abbreviations: OS, overall survival; SN, sentinel node; SNB, sentinel node biopsy.

*Two-sided $P$ value from Wald test.

†Third and first quartiles of age distribution, respectively.

$\ddagger$ Positive indicates undergoing SNB. 


\begin{tabular}{|c|c|c|c|c|c|c|c|c|c|c|c|c|c|}
\hline Points & 0 & 10 & 20 & 30 & 40 & & 50 & 60 & 70 & 80 & 90 & & 100 \\
\hline Age (years) & 15 & 20 & & 25 & 30 & & 35 & 40 & 45 & 5055 & 560 & 70 & 80 \\
\hline $\begin{array}{l}\text { Mitotic rate } \\
\left(\text { number } / \mathrm{mm}^{2}\right)\end{array}$ & & $\geq 1$ & & & & & & & & & & & \\
\hline Ulceration & No & & & Yes & & & & & & & & & \\
\hline $\begin{array}{l}\text { Lymphovascular } \\
\text { invasion }\end{array}$ & & Yes & & & & & & & & & & & \\
\hline Regression & No $/<\overline{50 \%}$ & & $\geq 5$ & & & & & & & & & & \\
\hline SLN status & $\mathrm{Neg}$ & & Pos & & & & & & & & & & \\
\hline Total points & 0 & 20 & 40 & 60 & 80 & & 00 & 120 & 140 & 160 & 180 & & 200 \\
\hline 12-year OS, no SLN & & & 0.99 & & 0.95 & 0.9 & 0.8 & 0.70 .60 .5 & 0.40 .30 .20 & 0.10 .050 .01 & & & \\
\hline 12-year OS, SLN & & & & 0.99 & & 0.95 & 0.9 & $\begin{array}{ll}0.8 & 0.7 \\
0.7\end{array}$ & & & $0.05 \quad 0.01$ & & \\
\hline
\end{tabular}

Fig 1. Nomogram for 12-year overall survival (OS) in thin cutaneous melanoma. To calculate the survival probability for a specific patient, locate patient age and draw a line straight upward to the Points axis to determine the score associated with that age. Repeat the process for mitotic rate, ulceration, lymphovascular invasion, regression, and sentinel node (SN) status (when sentinel node biopsy [SNB] was performed), sum the scores for each factor, and locate this sum on the Total Points axis. Then, depending on whether or not SNB was performed, draw a line straight down to the corresponding 12-year OS axis to find the predicted OS probability. SLN, sentinel lymph node. are also present in the subset not receiving SNB, which in turn influences survival.

The nomogram was internally validated by the calibration plot in Figure 2, and by computing the bootstrap-corrected Harrell C statistic. The calibration plot suggests that the nomogram was well calibrated; predicted and observed survival were in good agreement (circles lying almost directly on the reference line), with only minor discrepancies between observed (circles) and corrected-for-optimism (Xs) survival. A fairly high C statistic (0.88) was obtained, indicating good model discriminative ability.

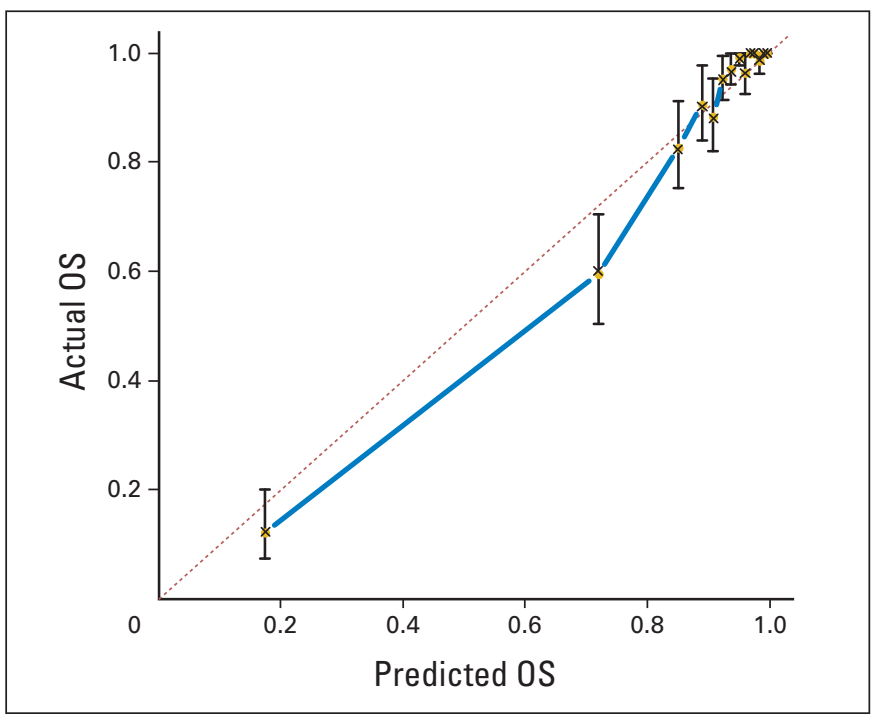

Fig 2. Calibration plots for internal validation of the overall survival (OS) nomogram. Nomogram-predicted probabilities were stratified in subgroups and, for each subgroup, the average predicted probability ( $x$ axis) was plotted against the Kaplan-Meier probability observed in the present case series ( $y$ axis). The $95 \% \mathrm{Cls}$ of the Kaplan-Meier estimates are indicated by vertical lines. The Xs represent observed survival corrected for optimism in the same subgroup. The dashed line is the reference line, indicating where an ideal nomogram would lie.

\section{DISCUSSION}

Indications for treating clinically node-negative melanoma $\leq 1 \mathrm{~mm}$ are continually being refined. ${ }^{3,9,10}$ One aim of this study was to investigate whether prognostic factors might predict regional lymph node involvement. We found that $M R \geq 1$, LVI in the primary tumor, or both were significantly associated with SN positivity. Among patients with metastatic SNs, MR $\geq 1$ or LVI were significantly more likely than MR less than 1 or no LVI $(P<.001$ in both cases). Previous studies have investigated MR as a determinant of SN status in smaller series. Kesmodel et $\mathrm{al}^{6}$ evaluated 181 patients with melanoma $\leq 1 \mathrm{~mm}$ undergoing SNB; they found that all patients with positive SNs also had MR more than 0 . Sondak et al ${ }^{18}$ developed a probabilistic model based on 419 patients with melanoma who had SNB. They found that patients younger than age 35 years with tumors less than $1 \mathrm{~mm}$ had a substantial risk of a positive SN, particularly if MR was high. Murali et $\mathrm{al}^{19}$ also found that LVI was associated with $\mathrm{SN}$ positivity in melanomas $\leq 1.0 \mathrm{~mm}$ and concluded that SNB should be considered in patients with lymphatic permeation of melanoma at the primary site.

We also found that Breslow thickness of more than $0.75 \mathrm{~mm}$ and tumor ulceration were significantly related to SN positivity; however, these variables are established independent predictors of SN status. ${ }^{3}$ Regarding regression, we found that the proportion of patients with a positive SN increased in the order of no regression, less than $50 \%$ regression, and $\geq 50 \%$ regression, but the association was not significant. Our findings therefore support the performance of SNB in patients with one or more of the following: more than $0.75 \mathrm{~mm}$ thickness, ulceration, MR one or more per square millimeter, and LVI.

Regarding outcomes, our findings provide support for the current melanoma staging system ${ }^{3}$ by showing that high MR and ulceration were significantly predictive of poorer survival.

In our multivariable analysis, SN status emerged as an independent factor predicting survival. However, SN status was not included 
as a prognostic factor in the most recent AJCC staging system, ${ }^{3}$ and the value of $\mathrm{SN}$ status has been debated. Han et $\mathrm{al}^{20}$ retrospectively evaluated 271 patients with melanomas $\leq 1 \mathrm{~mm}$ and showed that OS did not differ between patients with positive and negative SNs $(P=.53)$; however, this study was characterized by short follow-up (median 2.1 years). Venna et $\mathrm{al}^{21}$ examined SN status as a predictor of OS in 484 patients with thin melanoma, 34 of whom had a positive $\mathrm{SN}$; by multivariable analysis, $\mathrm{SN}$ status was the most powerful predictor of survival $(P=.009)$. That SN status predicts survival is in line with the idea that regional lymph node involvement is an indicator of the biologic aggressiveness of the disease and thus greater probability of extraregional spread and suggests that SN status can contribute to improving the risk stratification in patients with thin melanoma.

This study also found that LVI and extensive regression were independent predictors of OS, again in contrast to the current melanoma staging system. ${ }^{3} \mathrm{Xu}$ et $\mathrm{al}^{22}$ evaluated LVI as an independent prognostic factor in 251 patients with primary melanoma. Multivariable logistic regression for 10-year metastasis was used to define independent prognostic factors, from which a prognostic tree was developed to identify different risk groups. Among thin melanomas, the prognostic tree identified T1b melanomas with LVI as having poor prognoses. Egger et $\mathrm{al}^{23}$ investigated LVI in a cohort with primary melanoma of all thicknesses. They found that, although LVI was not an independent OS predictor, it was a powerful predictor of worse OS among patients with evidence of regression.

In our multivariable analysis, extensive regression $-\geq 50 \%$ of the entire lesion-was strongly associated with poor OS. Some studies have reported similar findings ${ }^{24,25}$; however, other studies have reported that regression has no effect on prognosis. ${ }^{26,27}$ It is possible that thin melanomas with regression are actually thicker, but that the measured thickness of residual melanoma in the regressed tumor is an underestimate of original thickness. In such cases, the metastatic potential of the tumor might be better predicted by the original thickness rather than the thickness of residual melanoma in the regressed tumor, thereby explaining the association of regression with metastasis. The effect of regression in masking thickness is likely to be proportionally greater in thin melanomas, a hypothesis supported by Massi et al, ${ }^{24}$ who found that tumor thickness and regression thickness were strong independent predictors of progression in thin melanomas.

On the basis of the hypothesis that the greater the extent of regression, the greater the underestimate of true thickness, we divided thin melanomas into two groups: one without regression or not exceeding $50 \%$, the other with regression $\geq 50 \%$. We found that in the latter group, regression was a reliable prognostic variable.

Because our series was large with long follow-up and strong prognostic associations were observed, we decided to develop a nomogram to predict 12-year OS. We propose the nomogram as a useful predictor of survival in individual patients and as a useful tool for risk stratification in clinical studies. It is noteworthy that the nomogram includes age as an important determinant of prognosis, because older age at diagnosis was significantly related to a poorer outcome. Other recent studies have also reported that older age was significantly associated with lower survival. ${ }^{28,29}$

Another interesting finding of our study was that most recurrences developed more than 5 years after diagnosis, and often 8 to 10 years later. In general, disease recurrence 10 years after initial treatment is rare; however, late recurrences are known, and they seem more common in patients with thin primary lesions. ${ }^{30}$

To conclude, we have found that LVI and extensive regression are independent predictors of survival in patients with thin melanomas, and we propose that these variables should be included in a revised melanoma staging system. We have also shown that SN status is an independent predictor of survival and that high MR and presence of LVI predict SN positivity, so their presence should suggest performing an SNB. In the absence of data from randomized controlled trials, our retrospective data provide rational bases for making treatment decisions in patients with thin melanoma by identifying those at relatively high risk of dying from their disease who may benefit from more aggressive treatments and long-term follow-up. Nevertheless, further studies on independent series are advisable to assess the reproducibility of our results, externally validate the nomogram, and confirm its utility for decision making.

\section{AUTHORS' DISCLOSURES OF POTENTIAL CONFLICTS OF INTEREST}

The author(s) indicated no potential conflicts of interest.

\section{AUTHOR CONTRIBUTIONS}

Conception and design: Andrea Maurichi, Rosalba Miceli, Tiziana Camerini, Luigi Mariani, Roberto Patuzzo, Giovanni Pellacani, Nicola Pimpinelli, Lorenzo Borgognoni, Daniele Bergamaschi, Catherine Harwood, Odysseas Zoras, Mario Santinami

Collection and assembly of data: Andrea Maurichi, Rosalba Miceli, Tiziana Camerini, Roberto Patuzzo, Roberta Ruggeri, Gianfranco Gallino, Elena Tolomio, Gabrina Tragni, Barbara Valeri, Andrea Anichini, Roberta Mortarini, Daniele Moglia, Giovanni Pellacani, Sara Bassoli, Caterina Longo, Nicola Pimpinelli, Lorenzo Borgognoni, Daniele Bergamaschi, Catherine Harwood, Odysseas Zoras, Mario Santinami Data analysis and interpretation: Rosalba Miceli, Tiziana Camerini, Luigi Mariani, Roberto Patuzzo, Roberta Ruggeri, Gianfranco Gallino, Elena Tolomio, Gabrina Tragni, Barbara Valeri, Andrea Anichini, Roberta Mortarini, Daniele Moglia, Giovanni Pellacani, Sara Bassoli, Caterina Longo, Pietro Quaglino, Nicola Pimpinelli, Lorenzo Borgognoni, Daniele Bergamaschi, Catherine Harwood, Odysseas Zoras, Mario Santinami

Manuscript writing: All authors

Final approval of manuscript: All authors

\section{REFERENCES}

1. Parkin DM, Ferlay J, Curado MP, et al: Fifty years of cancer incidence: $\mathrm{Cl} 5 \mathrm{I-IX}$. Int $\mathrm{J}$ Cancer 127:2918-2927, 2010

2. Gimotty PA, Elder DE, Fraker DL, et al: Identification of high-risk patients among those diag- nosed with thin cutaneous melanomas. J Clin Oncol 25:1129-1134, 2007

3. Balch CM, Gershenwald JE, Soong SJ, et al: Final version of 2009 AJCC melanoma staging and classification. J Clin Oncol 27:6199-6206, 2009

4. Balch CM, Murad TM, Soong SJ, et al: Tumor thickness as a guide to surgical management of clinical stage I melanoma patients. Cancer 43:883888, 1979

5. Kalady MF, White RR, Johnson JL, et al: Thin melanomas: Predictive lethal characteristics from a 30-year clinical experience. Ann Surg 238:528-535, 2003

6. Kesmodel SB, Karakousis GC, Botbyl JD, et al: Mitotic rate as a predictor of sentinel lymph node 
positivity in patients with thin melanomas. Ann Surg Oncol 12:449-458, 2005

7. Wong SL, Brady MS, Busam KJ, et al: Results of sentinel lymph node biopsy in patients with thin melanoma. Ann Surg Oncol 13:302-309, 2006

8. Yonick DV, Ballo RM, Kahn E, et al: Predictors of positive sentinel lymph node in thin melanoma. Am J Surg 201:324-327, 2011

9. Wong SL, Balch CM, Hurley $P$, et al: Sentinel lymph node biopsy for melanoma: American Society of Clinical Oncology and Society of Surgical Oncology joint clinical practice guideline. J Clin Oncol 30:2912-2918, 2012

10. Coit DG, Andtbacka R, Anker CJ, et al: Melanoma, version 2.2013: Featured updates to the NCCN guidelines. J Natl Compr Canc Netw 11:395407, 2013

11. Marubini E, Valsecchi MG: Analysing Survival Data for Clinical Trials and Observational Studies. Chichester, United Kingdom, John Wiley \& Sons, 1995

12. Gray RJ: A class of K-sample tests for comparing the cumulative incidence of a competing risk. Ann Stat 16:1141-1154, 1988

13. Schoenfeld D: Partial residuals for the proportional hazards regression model. Biometrika 69:239241, 1982

14. Akaike $\mathrm{H}$ : Information theory and an extension of the Maximum Likelihood principle, in Csáki F, Petrov BN (eds): Information Theory: Proceedings of the 2nd International Symposium. Budapest, Hungary, Akadémiai Kiedo, 1973

15. Harrell FE Jr, Lee KL, Mark DB: Multivariable prognostic models: Issues in developing models, evaluating assumptions and adequacy, and measuring and reducing errors. Stat Med 15:361-387, 1996

16. Efron B, Tibshirani RJ: An Introduction to the Bootstrap. New York, NY, Chapman and Hall, 1993

17. Durrleman S, Simon R: Flexible regression models with cubic splines. Stat Med 8:551-561, 1989

18. Sondak VK, Taylor JM, Sabel MS, et al: Mitotic rate and younger age are predictors of sentinel lymph node positivity: Lessons learned from the generation of a probabilistic model. Ann Surg Oncol 11:247-258, 2004

19. Murali R, Haydu LE, Quinn MJ, et al: Sentinel lymph node biopsy in patients with thin primary cutaneous melanoma. Ann Surg 255:128133, 2012

20. Han D, Yu D, Zhao $X$, et al: Sentinel node biopsy is indicated for thin melanomas $\geq 0.76 \mathrm{~mm}$. Ann Surg Oncol 19:3335-3342, 2012

21. Venna SS, Thummala $S$, Nosrati $M$, et al: Analysis of sentinel lymph node positivity in patients with thin primary melanoma. J Am Acad Dermatol 68:560-567, 2013

22. Xu X, Chen L, Guerry D, et al: Lymphatic invasion is independently prognostic of metastasis in primary cutaneous melanoma. Clin Cancer Res 18:229-237, 2012
23. Egger ME, Gilbert JE, Burton $A L$, et al: Lymphovascular invasion as a prognostic factor in melanoma. Am Surg 77:992-997, 2011

24. Massi D, Franchi $A$, Borgognoni $L$, et al: Thin cutaneous malignant melanomas $(<$ or $=1.5 \mathrm{~mm}$ ): Identification of risk factors indicative of progression. Cancer 85:1067-1076, 1999

25. McClain SE, Shada AL, Barry M, et al: Outcome of sentinel lymph node biopsy and prognostic implications of regression in thin malignant melanoma. Melanoma Res 22:302-309, 2012

26. Leiter U, Buettner PG, Eigentler TK, et al: Prognostic factors of thin cutaneous melanoma: An analysis of the central malignant melanoma registry of the German Dermatological Society. J Clin Oncol 22:3660-3667, 2004

27. Burton $A L$, Gilbert J, Farmer RW, et al: Regression does not predict nodal metastasis or survival in patients with cutaneous melanoma. Am Surg 77:1009-1013, 2011

28. Murali R, Haydu LE, Long GV, et al: Clinical and pathologic factors associated with distant metastasis and survival in patients with thin primary cutaneous melanoma. Ann Surg Oncol 19:17821789, 2012

29. Green AC, Baade $P$, Coory $M$, et al: Population-based 20-year survival among people diagnosed with thin melanomas in Queensland, Australia. J Clin Oncol 30:1462-1467, 2012

30. Faries MB, Steen $S, Y e X$, et al: Late recurrence in melanoma: Clinical implications of lost dormancy. J Am Coll Surg 217:27-34, 2013

\section{Affiliations}

Andrea Maurichi, Rosalba Miceli, Tiziana Camerini, Luigi Mariani, Roberto Patuzzo, Roberta Ruggeri, Gianfranco Gallino, Elena Tolomio, Gabrina Tragni, Barbara Valeri, Andrea Anichini, Roberta Mortarini, Daniele Moglia, Mario Santinami, Fondazione Istituto Di Ricovero e Cura a Carattere Scientifico (IRCCS), Istituto Nazionale dei Tumori, Milan; Giovanni Pellacani, Sara Bassoli, Caterina Longo, University Hospital of Modena and Skin Cancer Unit IRCCS Arcispedale Santa Maria Nuova, Reggio Emilia; Pietro Quaglino, University Hospital of Turin, Turin; Nicola Pimpinelli, Lorenzo Borgognoni, University Hospital of Florence and Istituto Tumori Toscano, S. Maria Annunziata Hospital, Florence, Italy; Daniele Bergamaschi, Catherine Harwood, Queen Mary University of London, London, United Kingdom; and Odysseas Zoras, University Hospital of Heraklion, Crete, Greece. 